ISSN 2072-6651

www.mdpi.com/journal/toxins

Article

\title{
In-vitro Neurotoxicity of Two Malaysian Krait Species (Bungarus candidus and Bungarus fasciatus) Venoms: Neutralization by Monovalent and Polyvalent Antivenoms from Thailand
}

\author{
Muhamad Rusdi Ahmad Rusmili ${ }^{1,2,3}$, Tee Ting Yee ${ }^{2}$, Mohd Rais Mustafa ${ }^{4}$, Iekhsan Othman ${ }^{2}$ \\ and Wayne C. Hodgson ${ }^{1, *}$
}

1 Monash Venom Group, Department of Pharmacology, Faculty of Medicine, Nursing and Health Sciences, Clayton, Victoria 3168, Australia; E-Mail: muhamad.rusmili@monash.edu

2 Jeffrey Cheah School of Medicine and Health Sciences, Monash University Sunway Campus, Bandar Sunway 46150, Malaysia; E-Mails: ting.tee@monash.edu (T.T.Y.);

iekhsan.othman@monash.edu (I.O.)

3 Department of Basic Medical Sciences, Kulliyyah of Pharmacy, International Islamic University Malaysia, Bandar Indera Mahkota 23800, Malaysia

4 Department of Pharmacology, Faculty of Medicine, University of Malaya, Kuala Lumpur 59100, Malaysia; E-Mail: rais@um.edu.my

* Author to whom correspondence should be addressed; E-Mail: wayne.hodgson@monash.edu; Tel.: +61-3-9905-4861; Fax: +61-3-9905-2547.

Received: 15 January 2014; in revised form: 28 February 2014 / Accepted: 7 March 2014 /

Published: 12 March 2014

\begin{abstract}
Bungarus candidus and Bungarus fasciatus are two species of krait found in Southeast Asia. Envenoming by these snakes is often characterized by neurotoxicity and, without treatment, causes considerable morbidity and mortality. In this study, the in vitro neurotoxicity of each species, and the effectiveness of two monovalent antivenoms and a polyvalent antivenom, against the neurotoxic effects of the venoms, were examined in a skeletal muscle preparation. Both venoms caused concentration-dependent inhibition of indirect twitches, and attenuated responses to exogenous nicotinic receptor agonists, in the chick biventer preparation, with $B$. candidus venom being more potent than $B$. fasciatus venom. SDS-PAGE and western blot analysis indicated different profiles between the venoms. Despite these differences, most proteins bands were recognized by all three antivenoms. Antivenom, added prior to the venoms, attenuated the neurotoxic effect of the venoms.
\end{abstract}


Interestingly, the respective monovalent antivenoms did not neutralize the effects of the venom from the other Bungarus species indicating a relative absence of cross-neutralization. Addition of a high concentration of polyvalent antivenom, at the $t_{90}$ time point after addition of venom, partially reversed the neurotoxicity of $B$. fasciatus venom but not $B$. candidus venom. The monovalent antivenoms had no significant effect when added at the $t_{90}$ time point. This study showed that $B$. candidus and B. fasciatus venoms display marked in vitro neurotoxicity in the chick biventer preparation and administration of antivenoms at high dose is necessary to prevent or reverse neurotoxicity.

Keywords: venom; krait; snake; neurotoxicity; antivenom; Bungarus fasciatus; Bungarus candidus

\section{Introduction}

Snakes from the Bungarus genus, commonly known as kraits, are nocturnal venomous snakes found in many parts of Asia. There are three species of Bungarus found in Malaysia: Bungarus candidus (Malayan krait), Bungarus fasciatus (banded krait) and Bungarus flaviceps (red-headed krait) [1]. There are two subspecies of Bungarus flaviceps, Bungarus flaviceps flaviceps and Bungarus flaviceps baluensis [2]. A high number of krait envenoming cases have been reported in India, Sri Lanka, Thailand and Vietnam [3-8]. However, envenoming by kraits is relatively uncommon in Malaysia [9,10].

Neurotoxicity characterizes systemic envenoming by kraits [11] and has been attributed to the presence of two major classes of neurotoxins in the venom, i.e., presynaptic and postsynaptic neurotoxins $[7,12,13]$. Presynaptic neurotoxins disrupt the release of the neurotransmitter acetylcholine from the nerve terminal while postsynaptic neurotoxins inhibit the binding of acetylcholine to the nicotinic receptors on the skeletal muscle [12]. Krait venoms have also been shown to contain acetylcholinesterase, hyalurodinase and L-amino acid oxidase, which have been hypothesized to enhance toxin penetration and contribute to the neurotoxic effect of the venoms [14].

Untreated respiratory muscle paralysis is the main cause of lethality in krait envenoming [3,15]. Morbidities in the form of permanent brain damage due to anoxia, cerebral ataxia, paraplesia, mydriasis, problems effecting micturation and causing constipation have also been reported in victims of krait bites $[4,16]$. Treatment options for systemic krait envenoming include administration of antivenom and supportive respiratory assistance. High doses of antivenom are often used in krait envenomings with limited success in reversing the neurotoxic symptoms [4,17]. The Queen Saovabha Memorial Institute (The Thai Red Cross Society; Bangkok, Thailand) produces B. fasciatus antivenom (BFAV) and B. candidus antivenom (BCAV) [18]. In addition, the Institute produces Neuro Polyvalent Snake antivenom (NPAV) for elapid envenoming which covers not only B. candidus and B. fasciatus but also Ophiophagus hannah and Naja kouthia [19].

The efficacy of NPAV against Malaysian elapid species and African cobras has been shown previously in in vivo experiments [19]. BFAV has been shown to have intraspecific neutralizing effects when tested against three species of krait found in Thailand [20]. However, BFAV was not effective when administered in patients envenomed by B. candidus [4]. BCAV reduced hospitalization time in 
patients envenomed by B. candidus in Thailand but there has been no study on the cross-neutralizing activity of this antivenom against other krait venoms [18]. The inability of polyvalent antivenoms, containing antibodies raised against the venoms of different Bungarus species, to neutralize neurotoxicity has been previously documented [5,21]. Geographical variations in venom composition have also been found to be a key factor in determining antivenom efficacy [22-24]. Pharmacological, biochemical and proteomic studies on various snake species showed that there are differences in the activities and composition of venom from the same snake species from different localities [23,25-27]

In the current study, the in vitro neurotoxic activity of Malaysian B. candidus and B. fasciatus venoms was assessed in the indirectly-stimulated chick biventer cervicis nerve-muscle preparation. The time to $90 \%$ inhibition of the original twitch height $\left(\right.$ i.e., $\left.t_{90}\right)$ was used to quantify the neurotoxicity of the venoms [28]. The neutralizing capability of two monovalent antivenoms, BCAV and BFAV, and a polyvalent antivenom, NPAV, against Malaysian B. candidus and B. fasciatus was also assessed. In addition, the cross-neutralising capability of the monovalent antivenoms was evaluated.

\section{Results}

\subsection{Chick Biventer Cercivis Nerve-Muscle Preparation}

B. candidus (1 and $10 \mu \mathrm{g} / \mathrm{mL}$; Figure 1a) and B. fasciatus (1 and $10 \mu \mathrm{g} / \mathrm{mL}$; Figure 2a) venoms abolished indirect twitches of the chick biventer cervicis nerve-muscle preparation. The time required for the twitches to be reduced by $90 \%$ (i.e., $t_{90}$ ) were: $B$. candidus venom; $1 \mu \mathrm{g} / \mathrm{mL}, 61 \pm 8 \mathrm{~min}$; $10 \mu \mathrm{g} / \mathrm{mL}, 13 \pm 4 \mathrm{~min}$ and for $B$. fasciatus venom; $1 \mu \mathrm{g} / \mathrm{mL}, 62 \pm 5 \mathrm{~min} ; 10 \mu \mathrm{g} / \mathrm{mL}, 22 \pm 5 \mathrm{~min}$. Repetitive washing with physiological salt solution, commencing at the $t_{90}$ time point after the addition of the venoms $(1 \mu \mathrm{g} / \mathrm{mL})$, did not reverse the neurotoxicity induced by either venom (data not shown).

B. candidus (1 and $10 \mu \mathrm{g} / \mathrm{mL}$; Figure $1 \mathrm{~b}$ ) and B. fasciatus (1 and $10 \mu \mathrm{g} / \mathrm{mL}$; Figure 2b) venoms almost completely abolished responses to acetylcholine (ACh) and carbachol (CCh) but not responses to potassium chloride $(\mathrm{KCl})$.

Figure 1. Effect of $B$. candidus venom on (a) indirect twitches of the chick biventer cervicis nerve-muscle preparation and (b) responses to exogenous agonists. $*: P<0.05$, significantly different from vehicle $(n=3-4$, one-way ANOVA for (a) and two-way ANOVA for (b)).
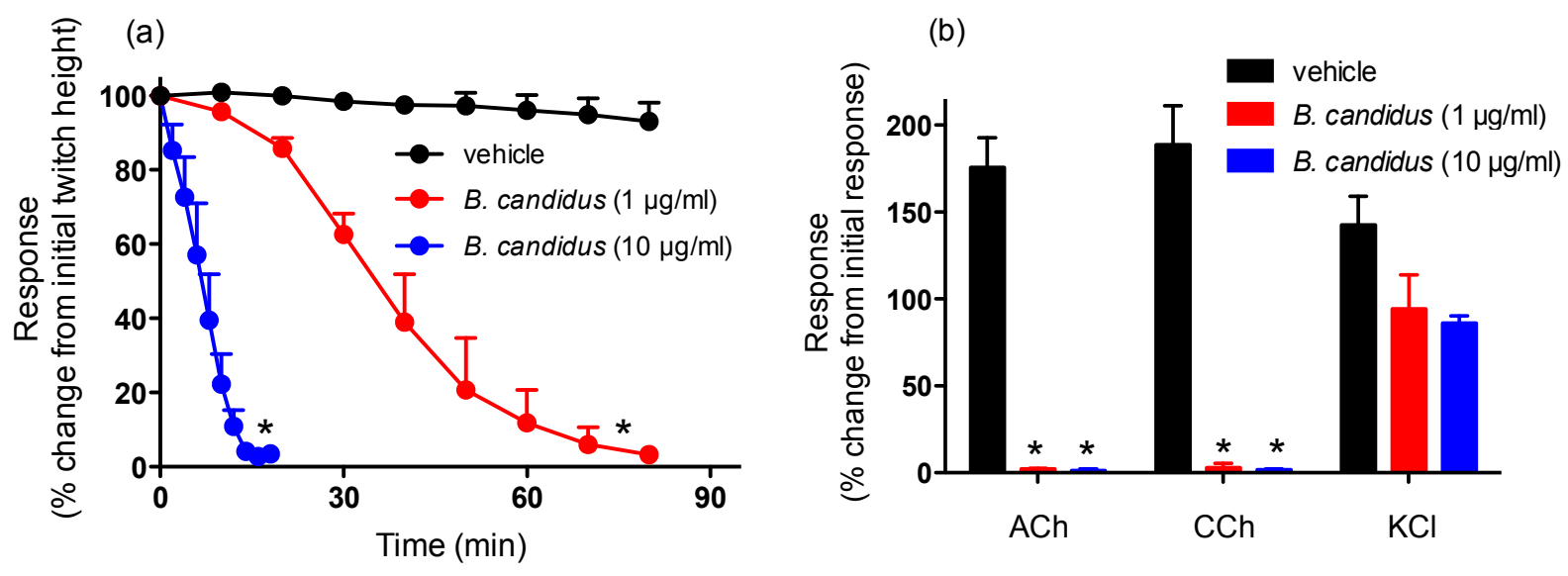
Figure 2. Effect of B. fasciatus venom on (a) indirect twitches of the chick biventer cervicis nerve-muscle preparation and (b) responses to exogenous agonists. $*: P<0.05$, significantly different from vehicle $(n=3-4$, one-way ANOVA for (a) and two-way ANOVA for (b)).
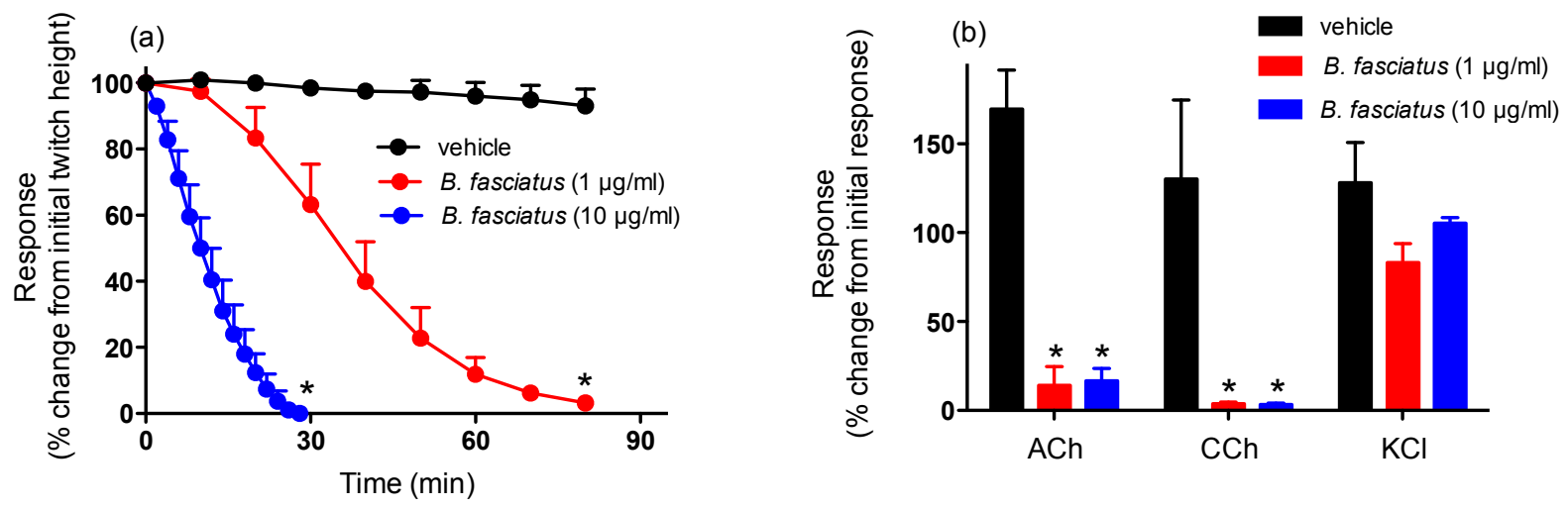

\subsection{Antivenom Studies}

Pre-incubation of monovalent $B$. candidus and $B$. fasciatus antivenoms at $1 \times, 3 \times$ or $5 \times$ the recommended titres (i.e., $1 \mathrm{~mL}$ per $0.6 \mathrm{mg}$ of $B$. fasciatus venom and $1 \mathrm{~mL}$ per $0.4 \mathrm{mg}$ of $B$. candidus venom) prior to the addition of $B$. candidus $(10 \mu \mathrm{g} / \mathrm{mL})$ or $B$. fasciatus $(10 \mu \mathrm{g} / \mathrm{mL})$ venoms, caused either a marked delay in the time to abolish twitches (i.e., $1 \times$ each antivenom) or prevented twitch inhibition (i.e., $3 \times$ and $5 \times$ concentrations of each antivenom) (Figure $3 \mathrm{a}, \mathrm{b}$ ).

Figure 3. Effect of prior addition of increasing concentrations of (a) B. candidus antivenom (BCAV) on the neurotoxic effects of $B$. candidus venom $(10 \mu \mathrm{g} / \mathrm{mL})$ or (b) $B$. fasciatus antivenom (BFAV) on the neurotoxic effects of $B$. fasciatus venom $(10 \mu \mathrm{g} / \mathrm{mL})$ in the chick biventer cervicis nerve-muscle preparation. ${ }^{*}: P<0.05$, significantly different than the respective venom alone ( $n=3-4$, one-way ANOVA).
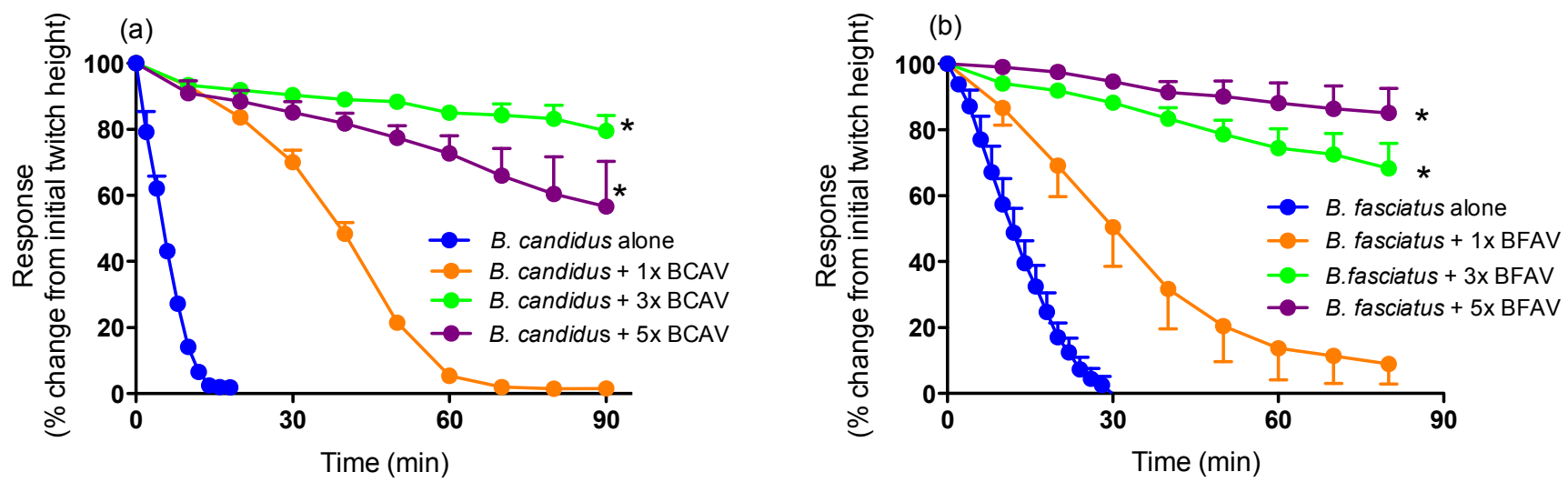

Pre-incubation with $3 \times$ the recommended titre of NPAV also markedly attenuated the reduction in twitch height induced by B. candidus $(10 \mu \mathrm{g} / \mathrm{mL})$ and B. fasciatus $(10 \mu \mathrm{g} / \mathrm{mL})$ venoms (Figure $4 \mathrm{a}, \mathrm{b})$. 
Figure 4. Effect of prior addition of increasing concentrations of Neuro Polyvalent antivenom (NPAV) on the neurotoxic effects of (a) B. candidus venom $(10 \mu \mathrm{g} / \mathrm{mL})$ or (b) B. fasciatus venom $(10 \mu \mathrm{g} / \mathrm{mL})$ in the chick biventer cervicis nerve-muscle preparation. *: $P<0.05$, significantly different than the respective venom alone ( $n=3-4$, one-way ANOVA).
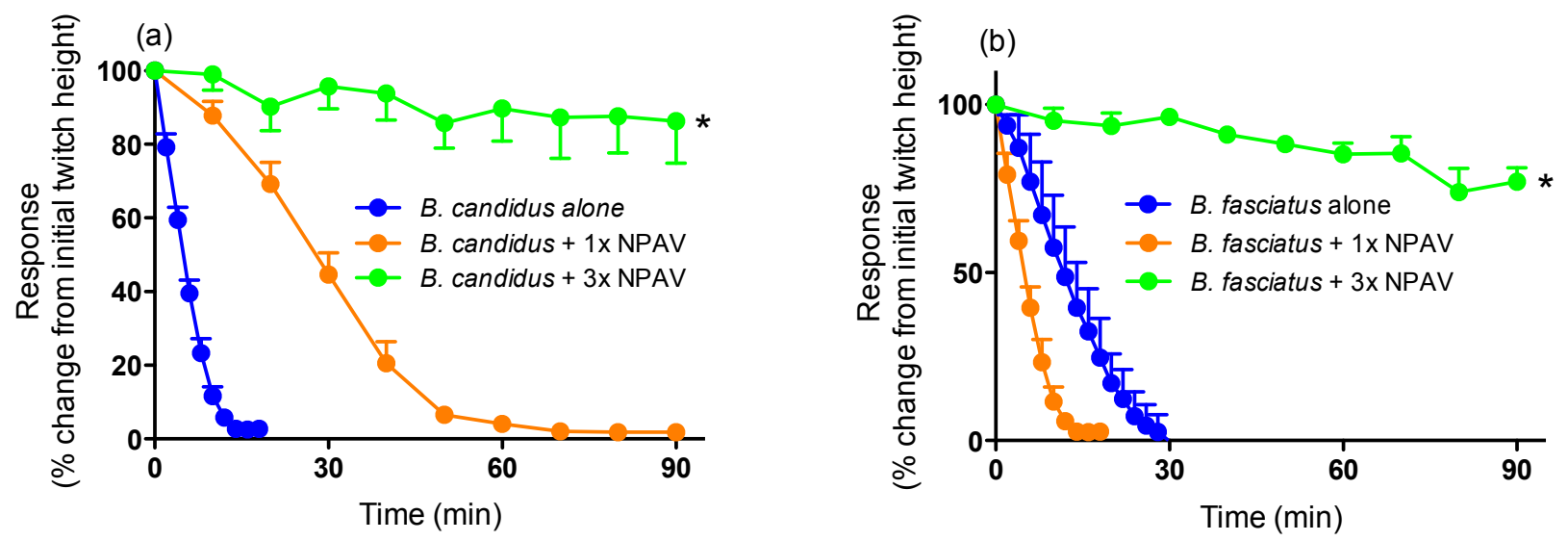

The prior addition of monovalent BFAV $(3 \times$ titre $)$ was unable to prevent the reduction in twitches induced by the subsequent addition of B. candidus venom $(10 \mu \mathrm{g} / \mathrm{mL})$ (Figure 5). Similarly, the prior addition of monovalent BCAV $(3 \times$ titre) was unable to prevent the reduction in twitches induced by the subsequent addition of $B$. fasciatus venom $(10 \mu \mathrm{g} / \mathrm{mL})$ (Figure 5$)$.

Figure 5. Effect of prior addition of $B$. candidus antivenom $(3 \times$; BCAV) or B. fasciatus antivenom (3×; BFAV) on the neurotoxic effects of $B$. fasciatus $(10 \mu \mathrm{g} / \mathrm{mL} ; n=3)$ or B. candidus $(10 \mu \mathrm{g} / \mathrm{mL} ; n=3)$ venoms, respectively, in the chick biventer cervicis nerve-muscle preparation.

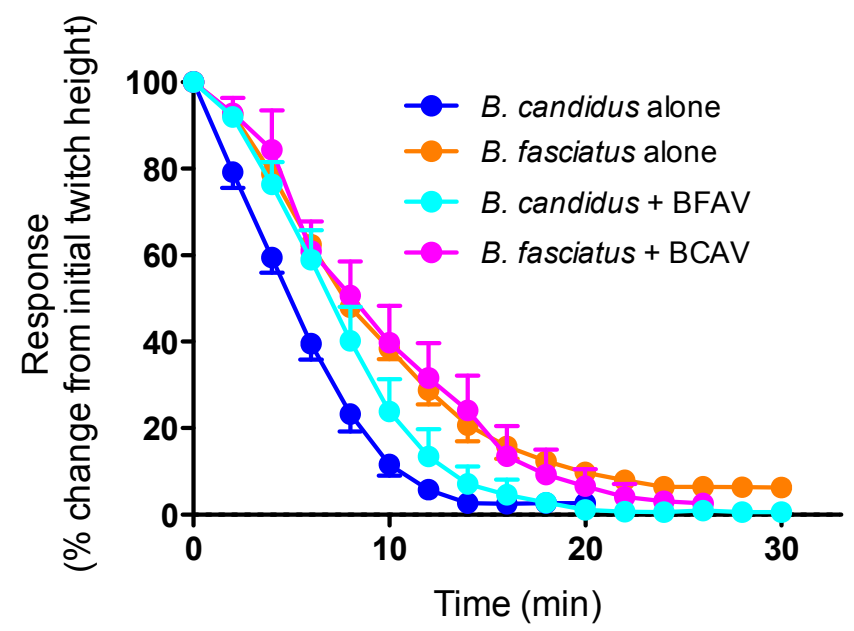

Neither monovalent antivenom $(3 \times$ recommended titre) significantly restored twitch height when added at the $t_{90}$ time point. However, NPAV produced a small but significant increase in twitch height for B. fasciatus venom when added at the $t_{90}$ time point i.e., approximately $20 \%$ recovery of twitch height (Figure 6b). 
Figure 6. Effect of (a) B. candidus antivenom (3×; BCAV) or Neuro Polyvalent antivenom ( $3 \times$; NPAV), added at the $t_{90}$ time point, on the neurotoxic effects of $B$. candidus (10 $\mu \mathrm{g} / \mathrm{mL})$ venom or (b) B. fasciatus antivenom (3×; BFAV) or Neuro Polyvalent antivenom $\left(3 \times\right.$; NPAV), added at the $t_{90}$ time point, on the neurotoxic effects of $B$. fasciatus $(10 \mu \mathrm{g} / \mathrm{mL})$ venom in the chick biventer cervicis nerve-muscle preparation. $*: P<0.05$, significantly different from zero, one sample t-test $(n=3)$.
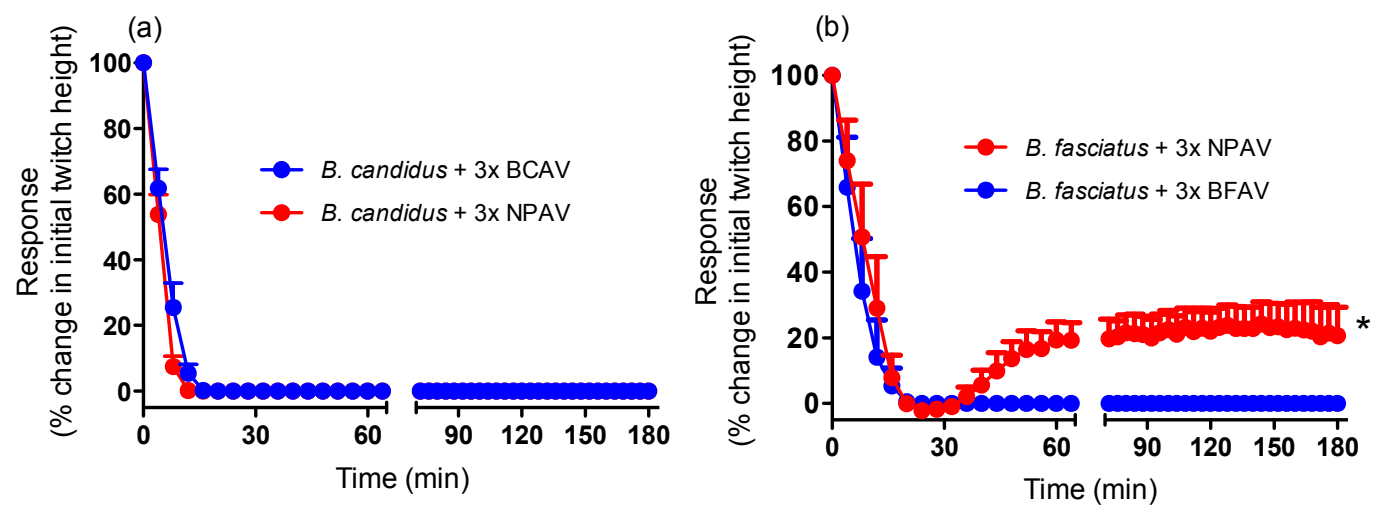

\subsection{SDS-PAGE and Western Blot}

SDS-PAGE analysis showed that B. candidus and B. fasciatus venoms consist of proteins of MW ranging from 10 to $75 \mathrm{kDa}$ (Figure 7). Non-reduced venoms possessed a greater number of protein bands compared to reduced venoms. Thick and high intensity bands clumped together were observed in the MW range of $10-15 \mathrm{kDa}$ in non-reduced $B$. candidus venom and $15-20 \mathrm{kDa}$ in non-reduced $B$. fasciatus venom. No protein band was observed within the range of $20-50 \mathrm{kDa}$ in reduced B. fasciatus venom. A single band was observed within the 150-250 kDa range in non-reduced B. fasciatus venom.

Figure 7. SDS-PAGE of venoms on a $12 \%$ gel. Venoms were treated in reducing and non-reducing buffer prior to loading and stained with Coomassie blue. $\mathrm{M}$ indicates the protein marker lane, $\mathrm{BC}(\mathrm{R})$ indicates $B$. candidus venom treated with reducing buffer and $\mathrm{BC}(\mathrm{NR})$ indicates $B$. candidus venom treated with non reducing sample buffer. $\mathrm{BF}(\mathrm{R})$ indicates $B$. fasciatus venom treated with reducing buffer and $\mathrm{BF}(\mathrm{NR})$ indicates $B$. fasciatus venom treated with non-reducing sample buffer.

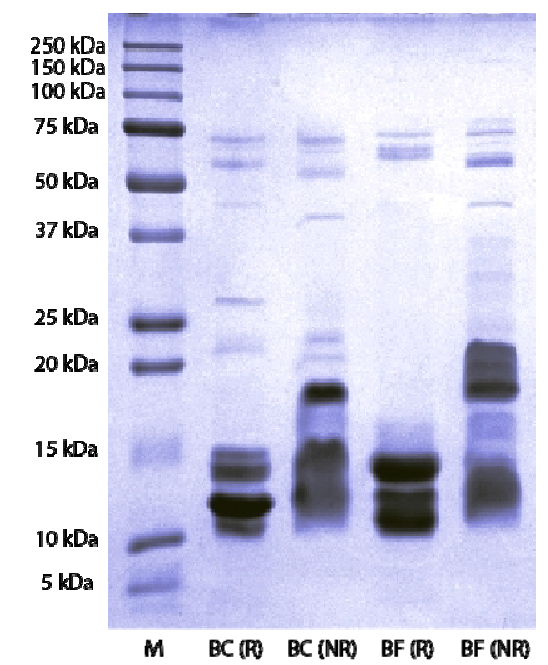


Western blotting of the gels with BCAV, BFAV or NPAV showed differences in the immunoreactivity profile against some protein bands in the venoms (Figure 8). Proteins belonging to the $15-50 \mathrm{kDa}$ and $100-150 \mathrm{kDa}$ ranges, previously not detected during SDS-PAGE, were identified by western blotting. The monovalent antivenoms were able to react with most of the protein bands from their respective species within the 15-20 kDa and 100-250 kDa weight ranges.

Figure 8. SDS-PAGE and western blot of venoms. $M$ indicates the protein marker lane, $\mathrm{BC}$ (B. candidus) and $\mathrm{BF}$ (B. fasciatus) indicate reduced venoms in 12\% gel. BCAV: reduced venoms incubated with monovalent $B$. candidus antivenom; BFAV: reduced venoms incubated with $B$. fasciatus antivenom; NPAV: reduced venoms incubated with neuro polyvalent antivenom. $\mathrm{X}$ indicates protein bands that were not detected based in the SDS-PAGE and in the membrane that was treated with the venom's monovalent antivenom.

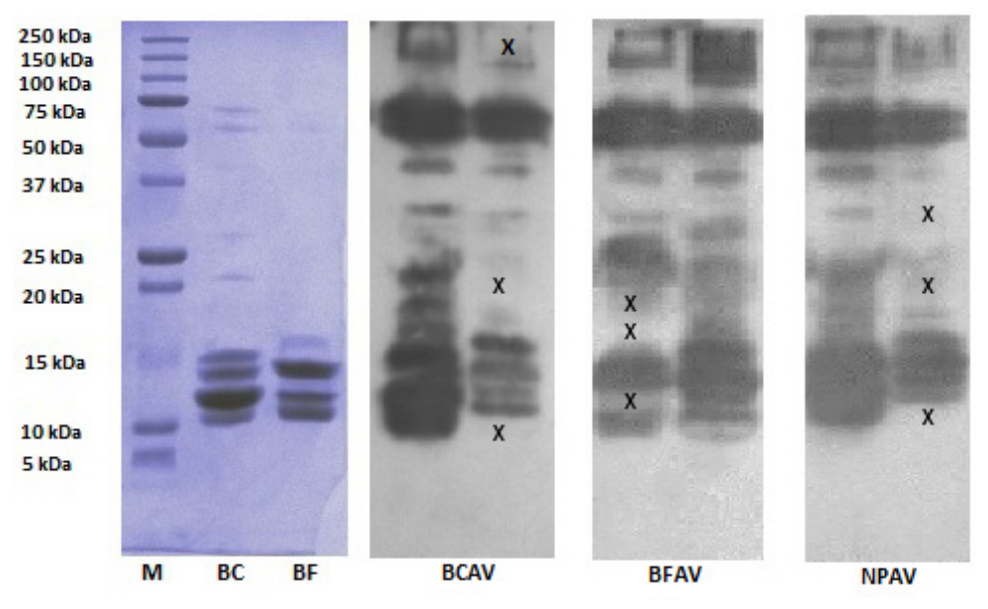

\section{Discussion}

This study showed that both $B$. candidus and $B$. fasciatus venoms cause marked in vitro neurotoxicity. Based on $t_{90}$ values, $B$. candidus was more potent than $B$. fasciatus venom. These studies are comparable to the results of previous lethality studies [14], although we caution against comparing the results of in vitro experiments to in vivo experiments. Contractile responses to ACh and $\mathrm{CCh}$, but not $\mathrm{KCl}$, were significantly attenuated by both venoms, indicating the likely presence of postsynaptic neurotoxins and an apparent lack of myotoxic activity. Repetitive washing with physiological salt solution did not reverse the neurotoxic effects of either venom, suggesting that the toxins inducing this effect were irreversible or pseudo-irreversible. It also supports the previous findings on the detection and isolation of potent presynaptic and postsynaptic neurotoxins in B. candidus and B. fasciatus venoms [7,15,29]. Pseudo-irreversible antagonism occurs when the agonist (e.g., acetylcholine released from the nerve terminal) and antagonist (e.g., post-synaptic neurotoxins in the venom) compete for the receptor (e.g., skeletal muscle nicotinic receptor) but the antagonist dissociates from the receptor so slowly (i.e., slow off-rate antagonist) that the agonist cannot achieve equilibrium with the receptor.

SDS-PAGE analysis showed that both venoms consist of a complex mixture of proteins with different molecular weights. The lower number of protein bands in reduced venoms compared to non-reduced venoms indicates the presence of proteins complexes in the venoms. Reduced presynaptic 
phospholipase and three-finger toxins in krait venoms are likely to be responsible for the higher number of bands within the 10-15 $\mathrm{kDa}$ range when the venoms are reduced $[13,30]$.

Western blotting against BCAV, BFAV and NPAV showed that there are differences in the immunoreactivity of protein components in the venoms. The absence of several bands in the blots indicate that not all proteins in the venoms react with antivenoms and these components may contribute to the lack of effectiveness of the antivenoms. However, a single band in SDS-PAGE may contain several proteins even after fractionation [31]. Therefore, there is a possibility that immunoreactive proteins may be masked with proteins that do not react with the antivenom within the same band. In addition, caution should be displayed when extrapolating the data from SDS-PAGE and western blot experiments as the bands correspond to denatured proteins and we cannot exclude the possibility that the antivenoms may recognize some proteins in vivo but not the western blot.

The efficacy of the antivenoms used in the current study have not previously been examined in vitro. Interestingly, a relatively high concentration (i.e., at least three times the recommended titre) of all antivenoms was required to prevent attenuation of indirect twitches. This may indicate that the composition of the Malaysian krait venoms are slightly different than the venoms used in the production of the antivenoms. Indeed, differences in the composition/activity of $B$. candidus and B. fasciatus venoms from different populations have been previously reported [7,32]. It is also possible that higher concentrations of antivenoms are required due to the lower immunogenicity of low molecular neurotoxins [22,33] which are responsible for the in vitro effects. The antivenoms were also largely ineffective, even at high concentrations, when added at the $t_{90}$ time point. The inability of the antivenoms to markedly reverse the neurotoxic effects, after venom administration, is likely to be related to the irreversible effects of presynaptic neurotoxins in the venoms [34].

The lack of effect of the monovalent antivenoms against the venom of the other krait species (i.e., BCAV against $B$. fasciatus venom, and vice versa) indicates a relative absence of cross-neutralization. This finding supports clinical observations where no beneficial outcome was seen when $B$. fasciatus antivenom was administered to patients envenomed by B. candidus [4] despite observed neutralization in animals [20]. The absence of cross-neutralization by either monovalent antivenom indicates that interspecies variation exists in the composition of the neurotoxins in the venoms. Therefore, it is possible that the immunoreactivity of some proteins observed in the western blot was due to binding of the antivenoms to invariant amino acid positions in the toxins [35] or at a position that had been modified during sample pretreatment [36]. Indeed, phospholipase $A_{2}$ neurotoxins and three finger toxin families are known to have similar invariant structural arrangements within the families [37,38].

\section{Experimental Section}

\subsection{Venoms and Antivenoms}

B. candidus and B. fasciatus venoms were a gift from Mr. Zainuddin Ismail (Perlis, Northwest Peninsular Malaysia). The snakes were collected in states of Kedah and Perlis, Northwest Peninsular Malaysia. The venoms from 10 snakes from each species were obtained and pooled by allowing snakes to bite plastic containers covered with Parafilm. After collection, the pooled venoms were transported on ice back to the laboratory, frozen at $-80{ }^{\circ} \mathrm{C}$ and then freeze-dried. Freeze-dried venom samples 
were weighed, labeled and stored at $-20{ }^{\circ} \mathrm{C}$ prior use. When required, the venoms were weighed and dissolved in Milli-Q water. B. candidus antivenom (Lot No.:BC00112) and B. fasciatus antivenom (Lot No.:BKC0108), and Neuro Polyvalent antivenom (Lot No.:KP00109) were purchased from Queen Saovabha Red Cross Society, Bangkok, Thailand. The freeze-dried antivenoms were dissolved with pharmaceutical grade water supplied by the manufacturer. The dissolved antivenoms were stored at $4{ }^{\circ} \mathrm{C}$ prior to use.

\subsection{Chemicals and Drugs}

The following were purchased from Sigma Aldrich (St. Louis, MO, USA): acetylcholine (ACh), carbamylcholine chloride (CCh), $d$-tubocurarine, bovine serum albumin.

\subsection{Protein Quantification by Bicinchoninic Acid Assay (BCA)}

Protein quantification was performed as instructed by the manufacturer (Pierce Biotechnology, Rockford, IL, USA). Approximately $25 \mu \mathrm{L}$ of sample or standard was loaded onto a 96-well plate in triplicate, then $200 \mu \mathrm{L}$ reagent buffer mix was added to each well. The plate was incubated at $37^{\circ} \mathrm{C}$ for $30 \mathrm{~min}$, then read at $562 \mathrm{~nm}$ using a plate reader spectrophotometer (VersaMax Elisa Microplate reader, Molecular Device, Sunnyvale, CA, USA). The protein concentration was determined from the standard curve.

\subsection{Indirectly-Stimulated Chick Biventer Cervicis Nerve-Muscle Preparation (CBCM)}

The experiments using this preparation were conducted using a method previously described $[21,23,35]$. Male chicks (5-12 days old) were killed by $\mathrm{CO}_{2}$ inhalation before the biventer nerve-muscle preparations were dissected and mounted in $5 \mathrm{~mL}$ organ baths aerated with carbogen $\left(5 \% \mathrm{CO}_{2}\right.$ and $\left.95 \% \mathrm{O}_{2}\right)$. The organ baths were filled with physiological salt solution of the following composition: $\mathrm{NaCl}, 118.4 \mathrm{mM}$; $\mathrm{KCl}, 4.7 \mathrm{mM} ; \mathrm{MgSO}_{4}, 1.2 \mathrm{mM} \mathrm{KH} \mathrm{PO}_{4}, 1.2 \mathrm{mM} ; \mathrm{CaCl}_{2}, 2.5 \mathrm{mM}$; $\mathrm{NaHCO}_{3}, 25 \mathrm{mM}$ and glucose, $11.1 \mathrm{mM}$. The tissues were stimulated every $10 \mathrm{~s}$ with pulses of $0.2 \mathrm{~ms}$ duration at a supramaximal voltage (10-20 V) using a Grass S88 stimulator attached to silver ring electrodes. Indirect stimulation of the tissues was confirmed by the abolishment of twitches by $d$-tubocurarine $(10 \mu \mathrm{M})$, which was then washed out until twitches were restored. In the absence of electrical stimulation, responses to acetylcholine (ACh; $1 \mathrm{mM}$ for $30 \mathrm{~s}$ ), carbachol (CCh; $20 \mu \mathrm{M}$ for $60 \mathrm{~s}$ ) and $\mathrm{KCl}$ (40 mM for $30 \mathrm{~s}$ ) were obtained. Responses were recorded on a PowerLab (ADInstruments, Bella Vista, NSW, Australia).

For antivenom studies, antivenom was added into the organ bath $10 \mathrm{~min}$ prior to the addition of venom or after the addition of venom at the $t_{90}$ time point.

\subsection{Sodium Dodecyl Sulphate Polyacylamide Gel Electrophoresis (SDS-PAGE)}

Sodium dodecyl sulphate polyacrylamide gel electrophoresis (SDS-PAGE) was conducted in 12\% polyacrylamide gel by using the method previously described [39]. The gels were then stained with Coomassie blue and de-stained by using 10\% acetic acid in water. Kaleidoscope Protein Marker (Bio-Rad, Hercules, CA, USA) was used in the gel for molecular weight protein marker. The gel was scanned using the ChemiDoc XRS Imaging System (BioRad, Hercules, CA, USA). 


\subsection{Western-Blot}

Venoms $(10 \mu \mathrm{g})$ were loaded and electrophoresed in 12\% separating SDS-PAGE gel with 5\% stacking gel as described in section 4.5. The venom was transferred onto polyvinylidene difluoride (PVDF) membrane (Merck Milipore, Billerica, MA, USA) by semi-dry electroblotting (BioRad, Hercules, CA, USA) at $10 \mathrm{~V}$ for $45 \mathrm{~min}$. The membrane was then blocked in Tris-buffered saline with $1 \%$ Tween 20 (TBST) buffer (20 mM Tris, $0.5 \mathrm{M} \mathrm{NaCl}$ and $0.5 \%$ Tween-20) supplemented with 5\% skim milk for $1 \mathrm{~h}$ at room temperature. Primary antibodies (i.e., Thai Red Cross Society's B. fascistus antivenom, B. candidus antivenom or Neuro Polyvalent antivenom diluted 1:500 fold in TBST with $5 \%$ skim milk) were then added and the membrane incubated overnight at $4{ }^{\circ} \mathrm{C}$. The membrane was then washed three times for 15 min with TBST buffer. Secondary antibody (i.e., goat anti-horse $\operatorname{IgG}$ secondary antibody conjugated with horseradish peroxidase (Santa Cruz Biotechnology Inc., Dallas, TX, USA) diluted in 1:5000 with TBST buffer with 5\% skim milk) was added to the membrane and left for $45 \mathrm{~min}$ at room temperature. The membrane was then washed three times for $10 \mathrm{~min}$ and the membrane incubated with Immobilon western chemiluminiscent (ECL) substrate (Merck Miliprore, Billerica, MA, USA) as described by the manufacturer. After incubation, the excess ECL reagent was removed and the membrane was exposed to x-ray film (Fujifim, Melbourne, Australia) to visualize the bands. The film and the membrane were scanned using the ChemiDoc XRS Imaging System (BioRad, Hercules, CA, USA).

\subsection{Data Analysis}

Changes in twitch height are expressed as a percentage of the original twitch height (i.e., prior to the addition of venom). Changes in the magnitude of contractile responses to exogenous agonists are expressed as a percentage of the original response to the agonist prior to the addition of venom. Data were analyzed by using a one-way analysis of variance (ANOVA) or two-way ANOVA followed by Dunnet's multiple comparison test (GraphPad Prism 6, La Jolla, CA, USA).

\section{Conclusions}

In conclusion, we found that Malaysian Bungarus candidus and Bungarus fasciatus venoms display marked in vitro neurotoxicity with $B$. candidus being slightly more potent than $B$. fasciatus. The neurotoxic effect of these venoms was attenuated by their respective monovalent antivenoms, albeit at higher concentrations than recommended, but there was limited cross neutralization. The apparent presence of potent, but low immunogenic neurotoxins, requires more investigation to identify methods to enhance the efficacy of the antivenoms. The data suggests that the non-specific Neuro Polyvalent antivenom may be useful for the treatment of B. candidus or B. fasciatus envenoming if the biting species cannot be determined or the specific monovalent antivenom is unavailable. Given the irreversible nature of the neurotoxic effects of the venoms, it would appear that early administration of the appropriate antivenom would be advantageous. A clinical trial is needed to assess the appropriate dose of antivenom to be use in the treatment for envenoming by these Malaysian species. This study also showed that there is a need for further work to explore geographical variation of the venoms from these species. 


\section{Acknowledgments}

We would like to acknowledge Zainuddin Ismail for the kind donation of Malaysian B. candidus and $B$. fasciatus venoms. This work was partially funded by a major research grant (fund number 5140068) from Monash University Sunway Campus, Malaysia and High Impact Research Grant (HIRG) (fund number E000002-20001) from the Ministry of Education (MOE), Government of Malaysia. Muhamad Rusdi Ahmad Rusmili is a recipient of SLAB scholarship from the Ministry of Higher Education, Government of Malaysia and is currently on study leave from the International Islamic University Malaysia, Malaysia.

\section{Conflicts of Interest}

The authors declare no conflict of interest.

\section{References}

1. Chanhome, L.; Cox, M.J.; Wilde H.; Jintakoon, P.; Chaiyabutr, N.; Sitprija, V. Venomous snakebite in Thailand. I: Medically important snakes. Mil. Med. 1998, 163, 310-317.

2. Kuch, U. Range extension and first record for Indonesia of the Kinabalu krait, Bungarus flaviceps baluensis LOVERIDGE, 1938. Herpatozoa 2002, 14, 149-151.

3. Hojer, J.; Tran Hung, H.; Warrell, D. Life-threatening hyponatremia after krait bite envenoming-A new syndrome. Clin. Toxicol. 2010, 48, 956-957.

4. Laothong, C.; Sitprija, V. Decreased parasympathetic activities in Malayan krait (Bungarus candidus) envenoming. Toxicon 2001, 39, 1353-1357.

5. Pochanugool, C.; Wilde, H.; Jitapunkul, S.; Limthongkul, S. Spontaneous recovery from severe neurotoxic envenoming by a Malayan krait Bungarus candidus (Linnaeus) in Thailand. Wilderness Environ. Med. 1997, 8, 223-225.

6. Pe, T.; Myint, T.; Htut, A.; Htut, T.; Myint, A.A.; Aung, N.N. Envenoming by Chinese krait (Bungarus multicinctus) and banded krait (B. fasciatus) in Myanmar. Trans. R. Soc. Trop. Med. Hyg. 1997, 91, 686-688.

7. Tsai, I.H.; Tsai, H.Y.; Saha, A.; Gomes, A. Sequences, geographic variations and molecular phylogeny of venom phospholipases and threefinger toxins of eastern India Bungarus fasciatus and kinetic analyses of its Pro31 phospholipases A2. FEBS J. 2007, 274, 512-525.

8. Ariaratnam, C.A.; Sheriff, M.R.; Theakston, R.D.G.; Warrell, D.A. Distinctive epidemiologic and clinical features of common krait (Bungarus caeruleus) bites in sri lanka. Am. J. Trop. Med. Hyg. 2008, 79, 458-462.

9. Zulkifli, A.; Hashim, M.; Khairul Anuar, A. Snake bites in Kelantan, Peninsular Malaysia. Trop. Biomed. 1995, 12, 1-4.

10. Chew, K.; Khor, H.; Ahmad, R.; Rahman, N. A five-year retrospective review of snakebite patients admitted to a tertiary university hospital in Malaysia. Int. J. Emerg. Med. 2011, 4, 41.

11. Kanchanapongkul, J. Neurotoxic envenoming following bites by the Malayan krait (Bungarus candidus). J. Med. Assoc. Thail. 2002, 85, 945-948. 
12. Kuch, U.; Molles, B.E.; Omori-Satoh, T.; Chanhome, L.; Samejima, Y.; Mebs, D. Identification of alpha-bungarotoxin (A31) as the major postsynaptic neurotoxin, and complete nucleotide identity of a genomic DNA of Bungarus candidus from Java with exons of the Bungarus multicinctus alpha-bungarotoxin (A31) gene. Toxicon 2003, 42, 381-390.

13. Khow, O.; Chanhome, L.; Omori-Satoh, T.; Ogawa, Y.; Yanoshita, R.; Samejima, Y.; Kuch, U.; Mebs, D.; Sitprija, V. Isolation, toxicity and amino terminal sequences of three major neurotoxins in the venom of Malayan krait (Bungarus candidus) from Thailand. J. Biochem. 2003, 134, 799-804.

14. Tan, N.H.; Ponnudurai, G. A comparative study of the biological properties of krait (genus Bungarus) venoms. Comp. Biochem. Physiol. C. 1990, 95, 105-109.

15. Yanoshita, R.; Ogawa, Y.; Murayama, N.; Omori-Satoh, T.; Saguchi, K.; Higuchi, S.; Khow, O.; Chanhome, L.; Samejima, Y.; Sitprija, V. Molecular cloning of the major lethal toxins from two kraits (Bungarus flaviceps and Bungarus candidus). Toxicon 2006, 47, 416-424.

16. Awasthi, R.; Narang, S.; Chowdhury, P.P. Cerebellar ataxia following snake bite. JAPI 2010, 58, 389-390.

17. Chieh-Fan, C.; Tzeng-Jih, L.; Wen-Chi, H.; Hua-Wei, Y. Appropriate antivenom doses for six types of envenomations caused by snakes in taiwan. J. Venom Anim. Toxins 2009, 15, 479-490.

18. Leeprasert, W.; Kaojarern, S. Specific antivenom for Bungarus candidus. J. Med. Assoc. Thail. 2007, 90, 1467-1476.

19. Leong, P.K.; Sim, S.M.; Fung, S.Y.; Sumana, K.; Sitprija, V.; Tan, N.H. Cross neutralization of Afro-Asian cobra and Asian krait venoms by a Thai polyvalent snake antivenom (Neuro Polyvalent Snake Antivenom). PLoS Negl. Trop. Dis. 2012, 6, e1672.

20. Chanhome, L.; Wongtongkam, N.; Khow, O.; Pakmanee, N.; Omori-Satoh, T.; Sitprija, V. Genus specific neutralization of Bungarus snake venoms by Thai Red Cross banded krait antivenom. J. Nat. Toxins 1999, 8, 135-140.

21. Ali, S.A.; Yang, D.C.; Jackson, T.N.; Undheim, E.A.; Koludarov, I.; Wood, K.; Jones, A.; Hodgson, W.C.; McCarthy, S.; Ruder, T.; et al. Venom proteomic characterization and relative antivenom neutralization of two medically important Pakistani elapid snakes (Bungarus sindanus and Naja naja). J. Proteomics 2013, 89, 15-23.

22. Chotwiwatthanakun, C.; Pratanaphon, R.; Akesowan, S.; Sriprapat, S.; Ratanabanangkoon, K. Production of potent polyvalent antivenom against three elapid venoms using a low dose, low volume, multi-site immunization protocol. Toxicon 2001, 39, 1487-1494.

23. Fry, B.G.; Wickramaratna, J.C.; Jones, A.; Alewood, P.F.; Hodgson, W.C. Species and regional variations in the effectiveness of antivenom against the in vitro neurotoxicity of death adder (Acanthophis) venoms. Toxicol. Appl. Pharm. 2001, 175, 140-148.

24. Fry, B.G.; Winkel, K.D.; Wickramaratna, J.C.; Hodgson, W.C.; Wüster, W. Effectiveness of snake antivenom: Species and regional venom variation and its clinical impact. Toxin Rev. 2003, $22,23-34$.

25. Shashidharamurthy, R.; Jagadeesha, D.; Girish, K.; Kemparaju, K. Variation in biochemical and pharmacological properties of Indian cobra (Naja naja naja) venom due to geographical distribution. Mol. Cell. Biochem. 2002, 229, 93-101. 
26. Skejić, J.; Hodgson, W.C. Population divergence in venom bioactivities of elapid snake Pseudonaja textilis: Role of procoagulant proteins in rapid rodent prey incapacitation. PLoS ONE 2013, 8, e63988.

27. Chang, H.C.; Tsai, T.S.; Tsai, I.H. Functional proteomic approach to discover geographic variations of king cobra venoms from Southeast Asia and China. J. Proteomics 2013, 89, 141-153.

28. Hodgson, W.C.; Wickramaratna, J.C. In vitro neuromuscular activity of snake venoms. Clin. Exp. Pharmacol. Physiol. 2002, 29, 807-814.

29. Karsani, S.A.; Othman, I. Isolation, complete amino acid sequence and characterization of a previously unreported post-synaptic neurotoxin-AlphaN3, from the venom of Bungarus candidus. Biochem. Biophys. Res. Commun. 2009, 389, 343-348.

30. Bon, C.; Saliou, B. Ceruleotoxin: Identification in the venom of Bungarus fasciatus, molecular properties and importance of phospholipase $\mathrm{A}_{2}$ activity for neurotoxicity. Toxicon 1983, 21, 681-698.

31. Malih, I.; Ahmad Rusmili, M.R.; Tee, T.Y.; Saile, R.; Ghalim, N.; Othman, I. Proteomic analysis of Moroccan cobra Naja haje legionis venom using tandem mass spectrometry. J. Proteomics 2014, 96, 240-252.

32. Chanhome, L.; Khow, O.; Puempunpanich, S.; Sitprija, V.; Chaiyabutr, N. Biological characteristics of the Bungarus candidus venom due to geographical variation. J. Cell Anim. Biol. 2009, 3, 93-100.

33. Chanhome, L.; Puempunpanich, S.; Omori-Satoh, T.; Chaiyabutr, N.; Sitprija, V. A pilot experiment for production of malayan krait antivenom: Immunization of rabbits with Bungarus candidus venom. J. Nat. Toxins 2002, 11, 353-356.

34. Prasarnpun, S.; Walsh, J.; Awad, S.; Harris, J. Envenoming bites by kraits: The biological basis of treatment-resistant neuromuscular paralysis. Brain 2005, 128, 2987-2996.

35. Kornhauser, R.; Isbister, G.K.; O’Leary, M.A.; Mirtschin, P.; Dunstan, N.; Hodgson, W.C. Cross-neutralisation of the neurotoxic effects of Egyptian cobra venom with commercial tiger snake antivenom. Basic Clin. Pharmacol. Toxicol. 2013, 112, 138-143.

36. Gutierrez, J.M.; Lomonte, B.; Leon, G.; Alape-Giron, A.; Flores-Diaz, M.; Sanz, L.; Angulo, Y.; Calvete, J.J. Snake venomics and antivenomics: Proteomic tools in the design and control of antivenoms for the treatment of snakebite envenoming. J. Proteomics 2009, 72, 165-182.

37. Kini, R.M.; Doley, R. Structure, function and evolution of three-finger toxins: Mini proteins with multiple targets. Toxicon 2010, 56, 855-867.

38. Kini, R.M. Excitement ahead: Structure, function and mechanism of snake venom phospholipase $\mathrm{A}_{2}$ enzymes. Toxicon 2003, 42, 827-840.

39. Laemmli, U.K. Cleavage of structural proteins during the assembly of the head of bacteriophage T4. Nature 1970, 227, 680-685.

(C) 2014 by the authors; licensee MDPI, Basel, Switzerland. This article is an open access article distributed under the terms and conditions of the Creative Commons Attribution license (http://creativecommons.org/licenses/by/3.0/). 